\title{
Complacency, panic, and the value of gentle rule enforcement in addressing pandemics
}

\author{
The impact of pandemics is magnified by the coexistence of two contradicting reactions to rare dire risks: panic \\ and the 'it won't happen to me' effect that hastens spread of the disease. We review research that clarifies the \\ conditions that trigger the two biases, and we highlight the potential of gentle rule enforcement policies that can \\ address these problematic conditions.
}

\section{Ido Erev, Ori Plonsky and Yefim Roth}

B ehavioural research highlights the existence of two suboptimal behavioural reactions to rare dire risks, both of which can affect the course of pandemics. The first is to overreact, which in the case of a pandemic can lead to panic buying and grocery stockpiling, as well as to extreme reactions by policy makers that might lead to economic destruction. The other is to under-react, which can hasten spread of the disease, deepen the disaster and lead to the collapse of health systems. To cope with the pandemic, policy-makers need to know that both types of reactions exist within the population. A survey of U.S adults in the midst of the coronavirus pandemic found that $31 \%$ think the public is over-reacting and $40 \%$ think the public is under-reacting ${ }^{1}$.

Previous analyses of the existence of contradicting reactions to rare risks focused on the description-experience gap ${ }^{2}$ : whereas people often plan to be careful when they are presented with a description of the risk, over time and experience they get complacent and start behaving as if they believe that 'it won't happen to $\mathrm{me}^{\text {'3 }}$. Public reaction to the coronavirus pandemic suggests, however, a more general pattern. It seems that many decision-makers chose to neglect the risk even after receiving descriptions of the devastating impact of the pandemic in China, while in certain settings people exhibited panic behaviour even after gaining experience. Here we highlight that the robustness of the contradicting reactions to rare risks can be the product of a tendency to rely on small samples of past experiences.

\section{Reliance on small samples and the bimodal reaction to rare risks} The assumption that people tend to rely on small samples of past experiences is the basic tenet in models that performed best in a series of competitions to predict human choice behavior ${ }^{4-6}$. It is based on the hypothesis that people tend to rely on similar past experiences ${ }^{7}$. The idea is that when people face a choice scenario, they implicitly recall a few similar choice scenarios from the past and estimate the value of each current choice option based on the average utility (for example, monetary rewards or time saved) this option generated in these few similar past experiences.

To illustrate the implications of reliance on small samples for the reaction to rare risks, we simulated the behaviour of individuals who rely on a sample of five random past experiences (Supplementary Information) in the choice tasks described in Fig. 1. The 'Reckless or Responsible' task (Fig. 1a) abstracts a repeated choice between behaving recklessly (for example, freely visiting persons at high risk during a pandemic) and behaving responsibly. One example of responsible behaviour involves managing a balance between physical distancing and economic activity; another example involves the use of smartphone contact tracing applications that reduce the risk of infecting others. Parameters of the task reflect the idea that reckless behaviour is better most of the time but also leads to a much larger loss when a rare event occurs (for example, infection). Although in our simulations behaving responsibly is the better option on average (i.e., has higher expected payoff), after some experience, most individuals choose to behave recklessly, as if they underweight the rare event (even if all individuals are prescribed to initially act responsibly). Notably, both rational individuals (i.e., decision-makers who choose the option that maximizes their expected payoff) and most individuals who rely on large samples do not exhibit such behaviour (Supplementary Table 1).

Although most of the time experience leads people to behave as if they believe the negative outcome will not happen to them,
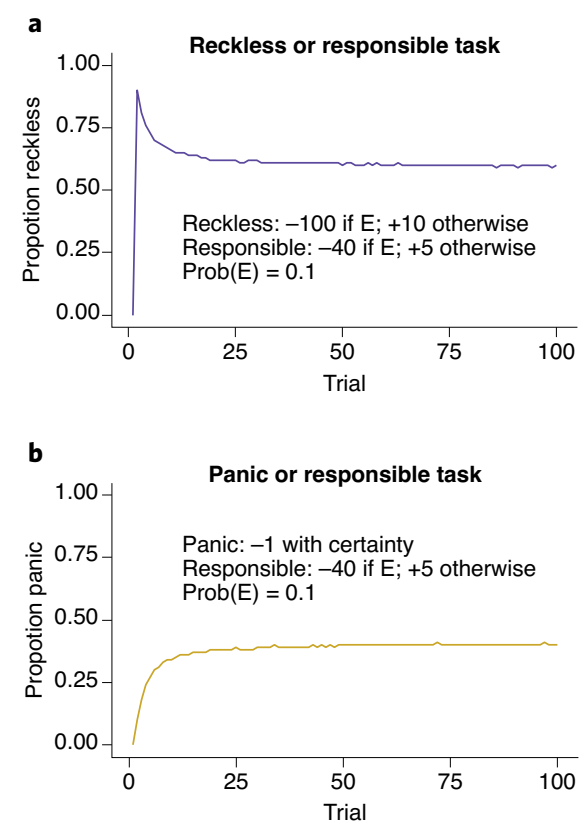

Fig. 1 | Simulations of two repeated-choice tasks. a, The emergence of the tendency to behave recklessly in the Reckless or Responsible task. b. The emergence of the tendency to exhibit panic in the Panic or Responsible task. E denotes the occurance of a rare event. Results reflect the choices of virtual decision-makers programmed to choose Responsible in the first trial and to base subsequent choices on a random sample of five past experiences (Supplementary Information). Recent choice prediction competitions show that assuming a reliance on small samples provides useful predictions of human behaviour.

in a significant minority of the cases people exhibit oversensitivity to rare disasters even after gaining experience. The 'reliance on small samples' hypothesis directly implies such a bimodal distribution of choices. Most small random samples are not likely to include any rare events, implying that the proportion of rare events in the sample 
is necessarily smaller than their objective probability. However, in a considerable minority of small samples, the proportion of rare events included in the sample exceeds their true probability. For example, $59 \%$ of the samples of size 5 drawn from a population with $10 \%$ rare disasters do not include any such disasters, but in the other $41 \%$ of samples, there is at least one disaster, suggesting the proportion of rare events in the sample is at least 0.2 , double the true objective proportion.

To see that, a considerable portion of the time, individuals who rely on small random samples behave as if they overweight rare events, consider the 'Panic or Responsible' task (Fig. 1b). This task can abstract a repeated choice between stockpiling groceries (Panic) and maintaining normal purchasing behaviour (Responsible). Panic prevents losses in case of long-term shortages of supplies but guarantees small storage and waste costs. Conversely, Responsible is better most of the time (avoiding the unnecessary small added costs), but carries a risk of a large loss in case of shortages. Notably, Responsible is also better on average and thus a choice of Panic reflects behaviour that appears as overweighting of the rare disaster. In our simulations, we assumed that all individuals are initially responsible; nevertheless, with experience, $40 \%$ of them learn to Panic. Again, neither rational decision-makers nor those who rely on large samples choose Panic so often (Supplementary Table 1).

\section{Contagious social behaviour}

In many natural settings, panic or reckless behaviour by some individuals increase the incentives of others to exhibit similar behaviours. For example, observing grocery stockpiling by others leads people to worry about the availability of grocery products in the near future. Consequently, they will engage in what appears to be panic shopping as well. Similarly, reckless behaviour of others in one's geographical vicinity or social group can lead to lockdowns in one's location or to stereotypical treatment of all individuals in one's group, reducing the benefits from adherence to the guidelines. Therefore, the existence of a minority of individuals who panic (or who are reckless) can make it more attractive for the majority to panic (or behave recklessly) as well. Such behavioural contagion has been well studied and validated in many other situations ${ }^{8-11}$. Additionally, it can be computationally shown to emerge in settings like those described in Fig. 1, even when some individuals in the population rely on large samples or are rational decision-makers.

\section{The potential of gentle rule enforcement}

Our discussion above suggests that people may not naturally demonstrate optimal behaviours towards a pandemic, even if it is in their best interest to do so. Authoritarian governments may choose to enforce responsible behaviours. One example of such enforcement comes from a policy, implemented in China, to harshly enforce use of smartphone contact tracing applications to control the spread of COVID-19. People had to use the recommended applications (and display a green signal on the application's screen) to enter stores and public facilities or to travel ${ }^{12}$. This policy helped break chains of transmission; when a user of the application was found to carry the virus, it was easy to identify users with whom the user recently interacted and isolate them to prevent secondary transmissions.

Notice that the harsh enforcement solution can be abstracted as a modification of the Reckless or Responsible task described above, in which the utility from Reckless is vastly reduced.

Although harsh enforcement policies of this kind can be very effective, they violate the rights of individuals and are incompatible with democratic principles. Our analysis suggests that it may be possible to design policies that are effective with more gentle enforcement that respects the rights of individuals ${ }^{13-15}$. To see why gentle rule enforcement policies can work, note that if people rely on small samples, they will not be sufficiently sensitive to rare (low probability) risks and instead will tend to base their decisions mostly on the common (high probability) experiences. For example, in the original Reckless or Responsible task, individuals that rely on small samples tend to behave recklessly because the common outcome from being Reckless $(+10)$ is better than the common outcome from being Responsible (+5), and most small samples include only these common outcomes (and no rare events). Thus, a gentle reduction of the common experience from reckless behaviour may be sufficient to eliminate the tendency to behave recklessly.

One way in which gentle rule enforcement can be implemented in natural settings involves interventions ensuring that, in most experiences, the responsible behaviour saves time and/or minimizes effort. In one demonstration of the value of this method, foremen in 11 Israeli factories were asked to encourage the use of safety devices by simply telling workers who did not use them to cease their current work and bring the missing safety devices. This gentle but frequent enforcement mechanism replaced a harsh one in which large fines were occasionally administered by the factories' safety inspectors. The results revealed a quick decrease, from $50 \%$ to $10 \%$, in safety rule violations ${ }^{15}$. Similar approaches could be implemented in the context of a pandemic. For example, to encourage the use of contact tracing applications, it may be enough to ask individuals that do not want to use the applications to fill longer forms and go through longer checks upon entry to public facilities. The effectiveness of gentle enforcement of the use of tracing applications is likely to also depend, of course, on the quality of the applications. Maximal effectiveness is expected when the application's false alarm rate is low and when the application is designed to respect individuals' privacy. Yet, insofar as these design aspects of the app do not contribute to the common outcomes of the decision to use or not use the application (i.e., if they only change the outcomes infrequently), their impact on these decisions may be smaller than one would expect.

The analysis of human reactions to experience leads to one discouraging and one encouraging prediction concerning the spread of pandemics. The discouraging prediction is that, without enforcement, many people may not behave optimally in response to the threat of a pandemic. The encouraging prediction is that enforcement does not have to be harsh: gentle reduction of the attractiveness of suboptimal behaviours can suffice.

\section{Ido Erev ${ }^{(\mathbb{D} 1 凶}$, Ori Plonsky ${ }^{(\mathrm{D})}{ }^{凶}$ and Yefim Roth (D) \\ ${ }^{1}$ Technion - Israel Institute of Technology, Haifa, Israel. ${ }^{2}$ University of Haifa, Haifa, Israel.

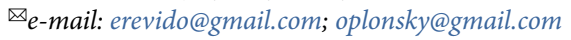

Published online: 14 August 2020 https://doi.org/10.1038/s41562-020-00939-z

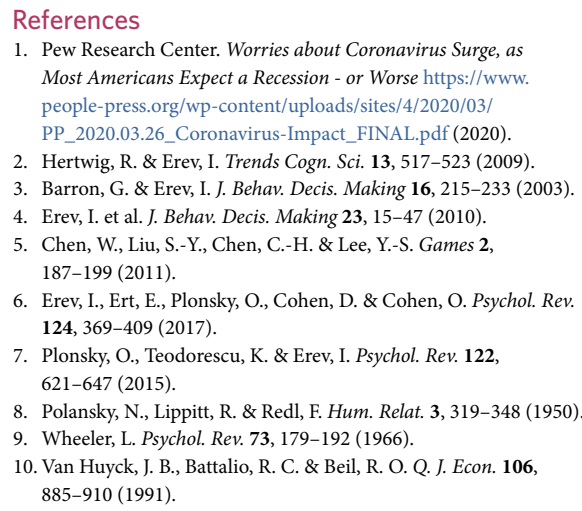

7. Plonsky, O., Teodorescu, K. \& Erev, I. Psychol. Rev. 122 621-647 (2015)

8. Polansky, N., Lippitt, R. \& Redl, F. Hum. Relat. 3, 319-348 (1950)

9. Wheeler, L. Psychol. Rev. 73, 179-192 (1966).

10. Van Huyck, J. B., Battalio, R. C. \& Beil, R. O. Q. J. Econ. 106 885-910 (1991). 
11. Christakis, N. A. \& Fowler, J. H. Stat. Med. 32, 556-577 (2013).

12. Xu Elegant, N. \& Chandler, C. When red is unlucky: what

we can learn from China's color-coded apps for tracking the coronavirus outbreak. Fortune https://fortune.com/2020/04/20/

china-coronavirus-tracking-apps-color-codes-covid-

19-alibaba-tencent-baidu/ (20 April 2020).

13. Erev, I., Ingram, P., Raz, O. \& Shany, D. Behav. Processes 84 366-371 (2010).

14. Nagin, D. S. Crime Justice 42, 199-263 (2013).

15. Schurr, A., Rodensky, D. \& Erev, I. J. Econ. Behav. Organ. 106 1-9 (2014).
Acknowledgements

We thank T. S. Wallsten for valuable comments on this manuscript. I.E. receives research funding from Israel Science Foundation: grant no. 535/17. The funder had no role in study design, data collection and analysis, decision to publish or preparation of the manuscript.

Author contributions

I.E., O.P. and Y.R. contributed to conceptualization of this paper. I.E. and O.P. performed the analysis and wrote the manuscript. I.E., O.P. and Y.R. reviewed and approved the final version.

Competing interests

The authors declare no competing interests.

Additional information

is available for this paper at https://doi.org/10.1038/ s41562-020-00939-Z. 